EGU2020-5608

https://doi.org/10.5194/egusphere-egu2020-5608

EGU General Assembly 2020

(c) Author(s) 2020. This work is distributed under

the Creative Commons Attribution 4.0 License.

\title{
SAR Imaging Geodesy with Electronic Corner Reflectors (ECR) and Sentinel-1 - First Experiences
}

\author{
Xanthi Oikonomidou ${ }^{1}$, Michael Eineder ${ }^{2}$, Christoph Gisinger ${ }^{2}$, Thomas Gruber ${ }^{1}$, Markus Heinze ${ }^{1}$, \\ and Vasiliki Sdralia ${ }^{1}$ \\ ${ }^{1}$ Institute of Astronomical and Physical Geodesy, Technical University of Munich, 80333 Munich, Germany \\ (xanthi.oikonomidou@tum.de) \\ ${ }^{2}$ Remote Sensing Technology Institute, German Aerospace Center, 82234 Weßling, Germany
}

SAR imaging geodesy is a new technique in the field of geodesy and remote sensing that enables the 3D localization of specifically designed radar targets. The absolute 3D position of a radar target in the ITRF can be estimated by means of least squares adjustment, when combining at least two sets of radar coordinates extracted from SAR images and the corresponding orbital arcs given by precise orbit determination. The installation of permanent radar targets allows for long-term position monitoring, making the technique a particularly interesting candidate for displacement and height change observations. While the principle of geodetic positioning with SAR is wellestablished, the selection of the radar target suitable for an application is subject to discussion. Parameters to be considered are the resolution of the radar images which can be provided by satellites like Sentinel-1 or TerraSAR-X, the size of the radar target with respect to the image resolution, the required localization accuracy of the selected application, and possible environmental and/or technical limitations at the installation site. Two main categories of artificial radar targets can be identified: passive reflectors and active transponders. Examples of passive reflectors that have been tested at geodetic observatories are corner reflectors, octahedron reflectors and tophats, while an example of active transponders is the experimental Electronic Corner Reflector (ECR).

The poster illustrates the first results acquired from the testing of ECRs operating in C-band, and their 3D localization using the IW medium resolution products of Sentinel-1A and 1B. The operation principle, the installation and mounting options, and the use of the ECRs as a small and portable alternative to passive reflectors are additionally discussed. 